\title{
Skin Prick Testing for Food Allergens in a Peripheral Metropolitan Unit
}

\author{
Rebecca Yingxue Qin a, b, Suganya Vignakaran ${ }^{\mathrm{a}, \mathrm{c}}$, Taren Bettler ${ }^{\mathrm{d}}$, Sarah Mulqueeny ${ }^{\mathrm{c}}$, \\ David Espinoza $^{\mathrm{e}}$, Stephen Sze Shing Teo ${ }^{\mathrm{a}}$, c, f, g
}

\begin{abstract}
Background: Skin prick testing (SPT) is a standard method of testing for allergies including food allergies. However, differentiation between allergy and sensitization requires clinical interpretation. Our aim was to describe the referral patterns and SPT results for food allergens in a cohort of children referred to a peripheral SPT unit at Mt Druitt Hospital, in Western Sydney.
\end{abstract}

Methods: This was a retrospective case record review.

Results: Over 1 year, 380 children were referred for SPTs. Of these, 295 had an SPT for at least one food allergen. Of these 295 children, 225 had a confirmed or suspected food allergy (FA), of whom 180 were positive on SPT for at least one food allergen. Children with asthma and/or eczema without a confirmed or suspected FA were less likely to have a positive SPT for food $(\mathrm{P}<0.001)$.

Conclusions: A child in this setting with a diagnosis of asthma and/or eczema in the absence of a clear diagnosis of FA is less likely to have a positive SPT for food.

Keywords: Skin prick testing; Food allergy; Asthma; Eczema

\section{Introduction}

Food-specific IgE testing comprises of skin prick testing (SPT)

Manuscript submitted August 27, 2021, accepted November 13, 2021

Published online December 31, 2021

aPaediatrics and Child Health, Western Sydney University, Sydney, NSW, Australia

bSydney Children's Hospital, Sydney, NSW, Australia

'Paediatrics Department, Blacktown and Mt Druitt Hospitals, Sydney, NSW, Australia

dWestmead Hospital, Corner Hawkesbury Road and Darcy Road, Westmead, Sydney, NSW, Australia

eNational Health and Medical Research Council (NHMRC) Clinical Trials Centre, University of Sydney, Sydney, NSW, Australia

fEmergency Department, Blacktown and Mt Druitt Hospitals, 75 Railway Street, Mt Druitt, Sydney, NSW, Australia

gCorresponding Author: Stephen Teo, Paediatrics Department, Mt Druitt Hospital, 75 Railway St, Mt Druitt, Sydney, NSW, Australia.

Email: s.teo@westernsydney.edu.au

doi: https://doi.org/10.14740/ijcp452 and radioallergosorbent testing (RAST). The advantages of SPT over RAST are the immediate result for both family and clinician and the avoidance of blood taking [1]. However, allergen selection, for example, tree nut panel, must be guided by the clinical history. The Royal Australasian College of Physicians in conjunction with the Australasian Society of Clinical Immunology and Allergy has recommended that "Food specific IgE testing should not be performed without a clinical history suggestive of IgE mediated food allergy" [2]. This is particularly relevant to our SPT service at Mt Druitt Hospital (MDH) which was, at that time, essentially a technical only service, with triaging of referrals, but no clinical assessment of the patients. Our hypotheses were that the majority of SPTs for food allergens in our setting were negative, and that the proportion of positive food allergen SPTs, defined as $>3 \mathrm{~mm}$, was higher when there was a suspected diagnosis of food allergy (FA), compared to other diagnoses such as asthma and eczema. Therefore, our aim was to describe the referral patterns and SPT results for food allergens in a cohort of children referred to our service.

\section{Materials and Methods}

The pediatric SPT service at MDH in metropolitan Western Sydney has pediatric nursing staff who are trained to administer but not necessarily interpret the results. Ordering and interpretation of SPTs are in the hands of referring clinicians, most of whom are general pediatricians, rather than our lead allergy clinician. SPT results and referring information were extracted from electronic case records for the year 2016. Ethics approval was granted by the Western Sydney Local Health District ethics committee (reference (5360) LNR/17/WMEAD/437). This study was conducted in compliance with the ethical standards of our institution on human subjects as well as with the Helsinki Declaration. A two-sided alpha of 5\% was applied to interpret the results of hypothesis tests. All statistical analysis was performed on SAS v9.4 (SAS Institute Inc., Cary, NC, USA).

\section{Results}

Overall, 380 children with a mean age of 4.7 years underwent SPTs in 2016. All 225 children with a known or suspected FA underwent an SPT for at least one food allergen. Of these 225 children, 36 also had eczema, nine also had asthma, and one had all three atopic diseases. In addition, children without a 
Table 1. Diagnoses of Children Referred for an SPT for Food

\begin{tabular}{llll}
\hline & Number of children & Number of children who had an SPT for food (\%) & P value \\
\hline FA & 225 & $225(100)$ \\
Asthma (without FA) & 42 & $29(69)$ \\
Eczema (without FA) & 59 & $49(83)$ \\
\hline
\end{tabular}

SPT: skin prick testing; FA: food allergy.

diagnosis of FA but with a diagnosis of asthma $(n=29)$ or eczema $(n=49)$ also underwent SPT for at least one food allergen (Table 1). In total, 225 children with clinical FA and 70 children without a known or suspected FA underwent an SPT for food (Fig. 1). Of the 225 children with clinical FA, 180 $(80 \%)$ were positive for at least one food allergen. Children referred for FA testing based on only a diagnosis of asthma or eczema, without FA, were unlikely to return a positive test $(\mathrm{P}<$ 0.001 , Table 2). Age was not related to the likelihood of a positive result to food allergens, with the mean of age of children with a positive SPT of 4.81 (standard deviation $(\mathrm{SD})=3.44$ ) years compared to $4.38(\mathrm{SD}=3.88)$ years for those who tested negative (Kruskal-Wallis test, $\mathrm{P}=0.61$ ) (Fig. 2).

\section{Discussion}

In this 1-year cohort, a child with a suspected or confirmed history of FA was more likely to have a positive SPT for food than a child with a history of asthma or eczema. However, a considerable proportion of children without a known or suspected FA were regardless referred for such testing. While we
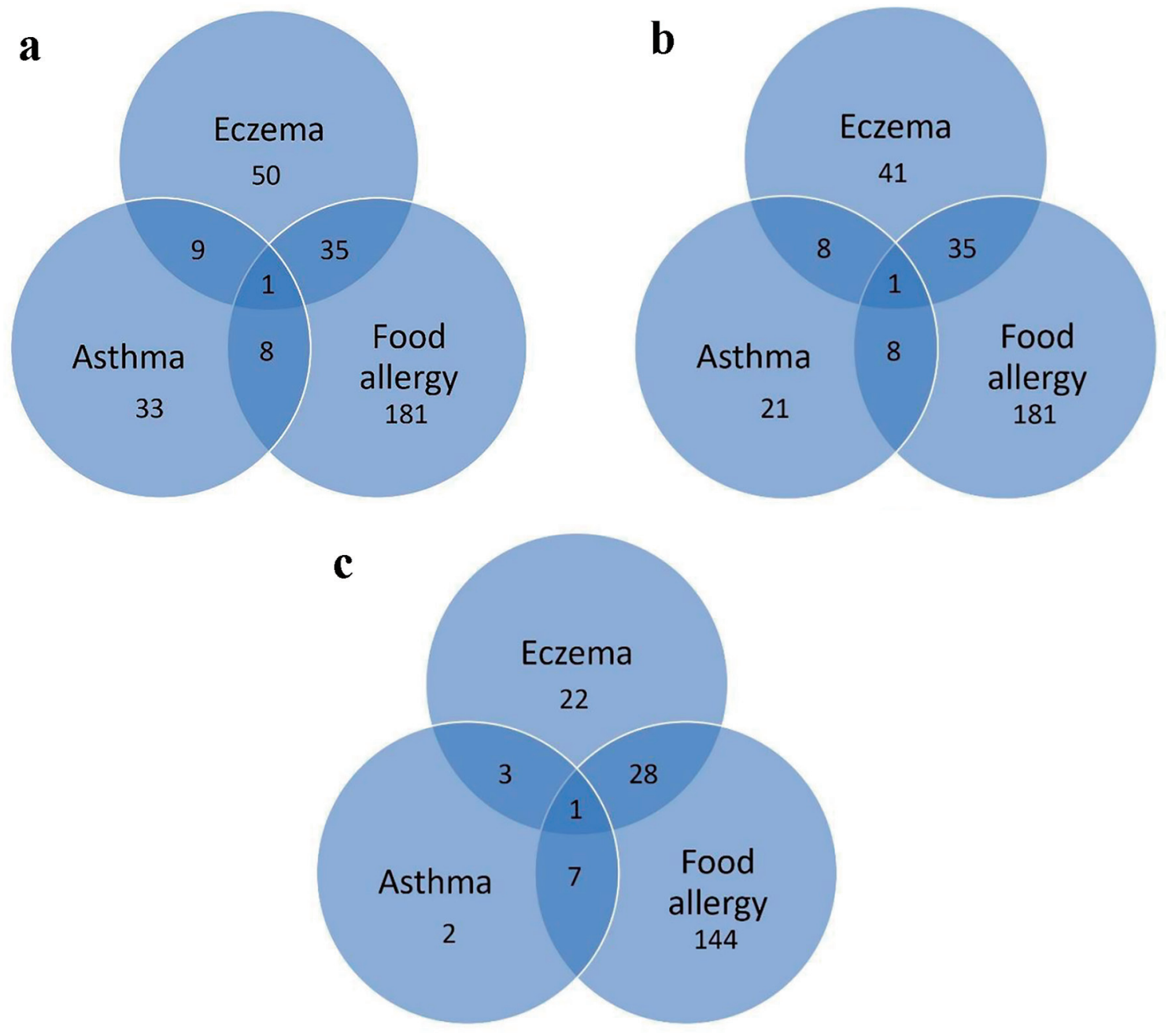

Figure 1. (a) Diagnoses of children referred for SPT. (b) Diagnoses of children referred for SPT for food. (c) Diagnoses of children with a positive SPT for food. SPT: skin prick testing. 
Table 2. SPT Results for Food

\begin{tabular}{lllll} 
& \multicolumn{3}{c}{ SPT } & Total (percent positive) \\
\cline { 2 - 4 } & Positive & Negative & $225(80)$ & P value \\
\hline FA & 180 & 45 & $29(17)$ \\
Asthma (without FA) & 5 & 24 & $49(51)$ \\
Eczema (without FA) & 25 & 24 & 0001 \\
\hline
\end{tabular}

SPT: skin prick testing; FA: food allergy.

suspected that some children with a negative SPT but a history of FA might be older and "growing out" of their allergy; our results did not support this hypothesis (Fig. 2).

The positive SPTs in these children without a history of FA may represent sensitization [3]. Clinicians must interpret SPT results in the context of a history that is, or is not, suggestive of a temporal relationship between a putative allergen and a perceived reaction. This process helps the clinician to recognize the difference between allergy and sensitization. Absence of this process, or family or child anxiety about a "positive test", may result in inappropriate dietary restriction with adverse nutritional and growth outcomes $[2,4]$. The prevalence of sensitization to food allergens is likely to be higher than the true prevalence of FA in our cohort. It has been suggested that SPTs are "slightly uncomfortable, but are usually well tolerated, even by small children" [1]. We would agree with this from the physical discomfort viewpoint. However, SPT, and particularly multiple allergen testing, may be psychologically stressful for child and family alike. It is our clinical experience that it can be particularly challenging to perform multiple pricks in younger children or in children with developmental and/or behavioral issues. These children can often become distressed and may require gentle but firm physical restraint similar to when a cannula is placed. Furthermore, a positive SPT to a food allergen requires a food challenge, to differentiate sensitization from allergy, particularly in children with atopic eczema $[5,6]$.

We are not implying that the referring clinicians are overordering SPTs. Rather there are possible valid reasons for such a high negative SPT rate. Firstly, the referring clinicians are mainly specialist pediatricians, thus, it is likely that a proportion of their clinical practice will comprise more severe eczema, asthma, or possible FAs. There may also be a higher degree of carer anxiety around the perceived severity of these diagnoses. Eliminating the possible role of any putative allergic trigger, even though the likelihood of one has been assessed as low or negligible, may be warranted when the alternative is a change or step-up in pharmacological management, particularly with higher dose inhaled corticosteroids or combined long-acting beta-2 agonists for moderate to severe asthma. Certainly, children with both asthma and FA are at a higher risk of adverse outcomes including mortality [7-9]. Additionally, and possibly more importantly, fear and anxiety related to allergies, real or imagined, particu-

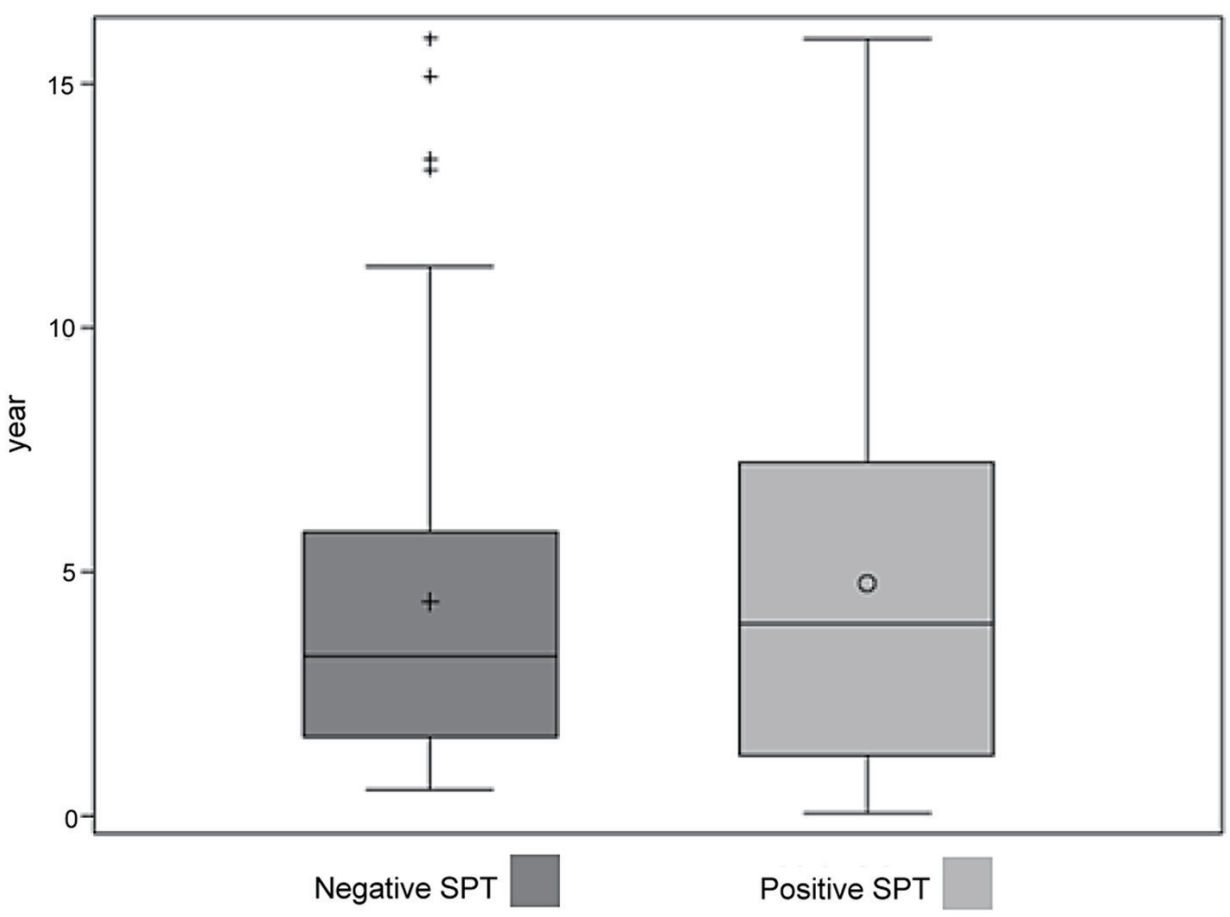

Figure 2. Ages of children with negative and positive SPT results to food allergens. SPT: skin prick testing. 
larly FAs, are well described [10-15]. Families of these children may have heightened anxiety about both the atopic disease and the concern or fear (or hope) that there is, or are, allergic triggers.

The goal is more selectivity in the number of children referred for testing and in the choice of SPT for each child. In turn, there will be less anxiety and discomfort for children undergoing SPTs, as well as savings in reagents and staff time and costs. Possible strategies for a more selective approach to referrals for SPTs for food allergens may be promoted through continuing professional development interventions, which may lead to a change in clinical practice [16], including more rational investigation ordering [17]. Feeding back our results to the referring practitioners, with peer review at the local lev$\mathrm{el}$, is a feasible intervention to change clinical behavior [18].

A limitation of this study is that we did not have access to full records of these children to confirm the clinical picture of FA, only referral diagnoses or very brief symptomatology on our request forms. However, most of our referral clinicians are general pediatric specialists so we accepted their diagnoses for this study.

We strongly suspect that referring clinicians are already counseling families regarding rationalization of testing, but that despite these efforts, some may only be convinced by a negative SPT, in which case, testing might be considered beneficial [19]. Therefore, we also plan to explore the experiences and approaches of the referring clinicians for children with possible FAs. Similarly, exploration of the expectations and attitudes of carers of these children referred for FA testing in our setting will be valuable.

\section{Conclusion}

A child with a diagnosis of eczema or asthma, but without a suspected or confirmed FA, was less likely to have a positive SPT for food than children with a suspected or known FA. However, these children made up a large proportion of referrals. Further work is required to explore clinicians' approaches and parental concerns for such children.

\section{Acknowledgments}

None to declare.

\section{Financial Disclosure}

None to declare.

\section{Conflict of Interest}

None to declare.

\section{Informed Consent}

Not applicable.

\section{Author Contributions}

Dr Rebecca Yingxue Qin: data collection and interpretation, literature review, and manuscript write-up. Dr Suganya Vignakaran: initial concept for the study, data interpretation, and review of manuscript write-up. Dr Taren Bettler: data collection and review of manuscript write-up. Ms Sarah Mulqueeny: review of manuscript write-up. Mr David Espinoza: statistical analysis. Prof Stephen Sze Shing Teo: initial concept for the study, data interpretation, and review of manuscript write-up.

\section{Data Availability}

Any inquiries regarding supporting data availability of this study should be directed to the corresponding author.

\section{Abbreviations}

SPT: skin prick testing; RAST: radioallergosorbent testing; FA: food allergy

\section{References}

1. The Australasian Society of Clinical Immunology and Allergy. Allergy testing [Internet]. 2015 [Accessed Sep 28, 2020]. Available at: https://allergy.org.au/images/pcc/ ASCIA_PCC_Allergy_testing_2020.pdf.

2. Australian Society of Clinical Immunology and Allergy. Top 5 low-value practices and interventions. 2017 [Accessed Oct 15, 2017]. Available at: https://evolve.edu. $\mathrm{au} /$ docs/default-source/logos/evolve_top-five_ascia. pdf?sfvrsn $=6$.

3. Allen KJ, Dharmage SC. The role of food allergy in the atopic march. Clin Exp Allergy. 2010;40(10):1439-1441.

4. Banerjee T, Banerjee A. Increased parental anxiety and voluntary allergenic food avoidance (VAFA) in the siblings of the index case (IC) with single or multiple food allergies: exploring the parental response to the interventions for addressing their anxiety and successful introduction of new food. Clin Transl Allergy. 2017;7(1):PD09.

5. Peters RL, Allen KJ, Dharmage SC, Koplin JJ, Dang T, Tilbrook KP, Lowe A, et al. Natural history of peanut allergy and predictors of resolution in the first 4 years of life: A population-based assessment. J Allergy Clin Immunol. 2015;135(5):1257-1266 e1251-1252.

6. Verduci E, Bianchi A, Brambilla M, Calvani M. Egg introduction during complementary feeding according to allergic risk: not just for peanuts! Ital J Pediatr. 2018;44(1):77.

7. Caffrey Osvald E, Bower H, Lundholm C, Larsson H, Brew BK, Almqvist C. Asthma and all-cause mortality in children and young adults: a population-based study. Thorax. 2020;75(12):1040-1046.

8. Di Palmo E, Gallucci M, Cipriani F, Bertelli L, Gianetti A, Ricci G. Asthma and food allergy: which risks? Me- 
dicina (Kaunas). 2019;55(9):509.

9. Pouessel G, Turner PJ, Worm M, Cardona V, Deschildre A, Beaudouin E, Renaudin JM, et al. Food-induced fatal anaphylaxis: From epidemiological data to general prevention strategies. Clin Exp Allergy. 2018;48(12):15841593.

10. Hoehn JL, Dahlquist LM, Hahn AL, Bollinger ME. Parents of children with food allergy: gender differences in perceived impact and perceived food allergy severity. J Pediatr Psychol. 2017;42(2):186-197.

11. Cummings AJ, Knibb RC, Erlewyn-Lajeunesse M, King RM, Roberts G, Lucas JS. Management of nut allergy influences quality of life and anxiety in children and their mothers. Pediatr Allergy Immunol. 2010;21(4 Pt 1):586594.

12. Antolin-Amerigo D, Manso L, Caminati M, de la Hoz Caballer B, Cerecedo I, Muriel A, Rodriguez-Rodriguez $\mathrm{M}$, et al. Quality of life in patients with food allergy. Clin Mol Allergy. 2016;14:4.

13. Petrovic-Dovat L, Fausnight T, White AM, Zeiger T, Bansal PS, Garg N, Annapareddy J, et al. Degree of anxiety in food allergic children in a tertiary care center. Ann Allergy Asthma Immunol. 2016;116(6):528-532.
14. Moen OL, Opheim E, Trollvik A. Parents experiences raising a child with food allergy; A qualitative review. J Pediatr Nurs. 2019;46:e52-e63.

15. Stensgaard A, Bindslev-Jensen C, Nielsen D. Peanut allergy as a family project: social relations and transitions in adolescence. J Clin Nurs. 2017;26(21-22):3371-3381.

16. Forsetlund L, O'Brien MA, Forsen L, Reinar LM, Okwen MP, Horsley T, Rose CJ. Continuing education meetings and workshops: effects on professional practice and healthcare outcomes. Cochrane Database Syst Rev. 2021;9:CD003030.

17. Morgan S, Coleman J. We live in testing times - teaching rational test ordering in general practice. Aust Fam Physician. 2014;43(5):273-276.

18. van Braak M, Visser M, Holtrop M, Statius Muller I, Bont J, van Dijk N. What motivates general practitioners to change practice behaviour? A qualitative study of audit and feedback group sessions in Dutch general practice. BMJ Open. 2019;9(5):e025286.

19. Tang MLK, Koplin JJ, Sampson HA. Is skin testing or sIgE testing necessary before early introduction of peanut for prevention of peanut allergy? J Allergy Clin Immunol Pract. 2018;6(2):408-413. 